\title{
Die äußere Wendung ist einen Versuch wert
}

\section{Die äußere Wendung wird bei Beckenendlage ab der 36. SSW von internationalen Gesellschaften empfohlen, um eine vaginale Geburt zu begünstigen. Eine Arbeitsgruppe an der Charité Universitätsklinik Berlin erforscht derzeit, welche Kriterien mit einem erfolgreichen Wendungsversuch korrelieren. Dr. Larry Hinkson berichtet auf dem Forum Hebammenarbeit 2020 in Leipzig und Wiesbaden, wie die Ergebnisse helfen, schwangere Frauen besser über Chancen und Risiko von Wendungen zu beraten.}

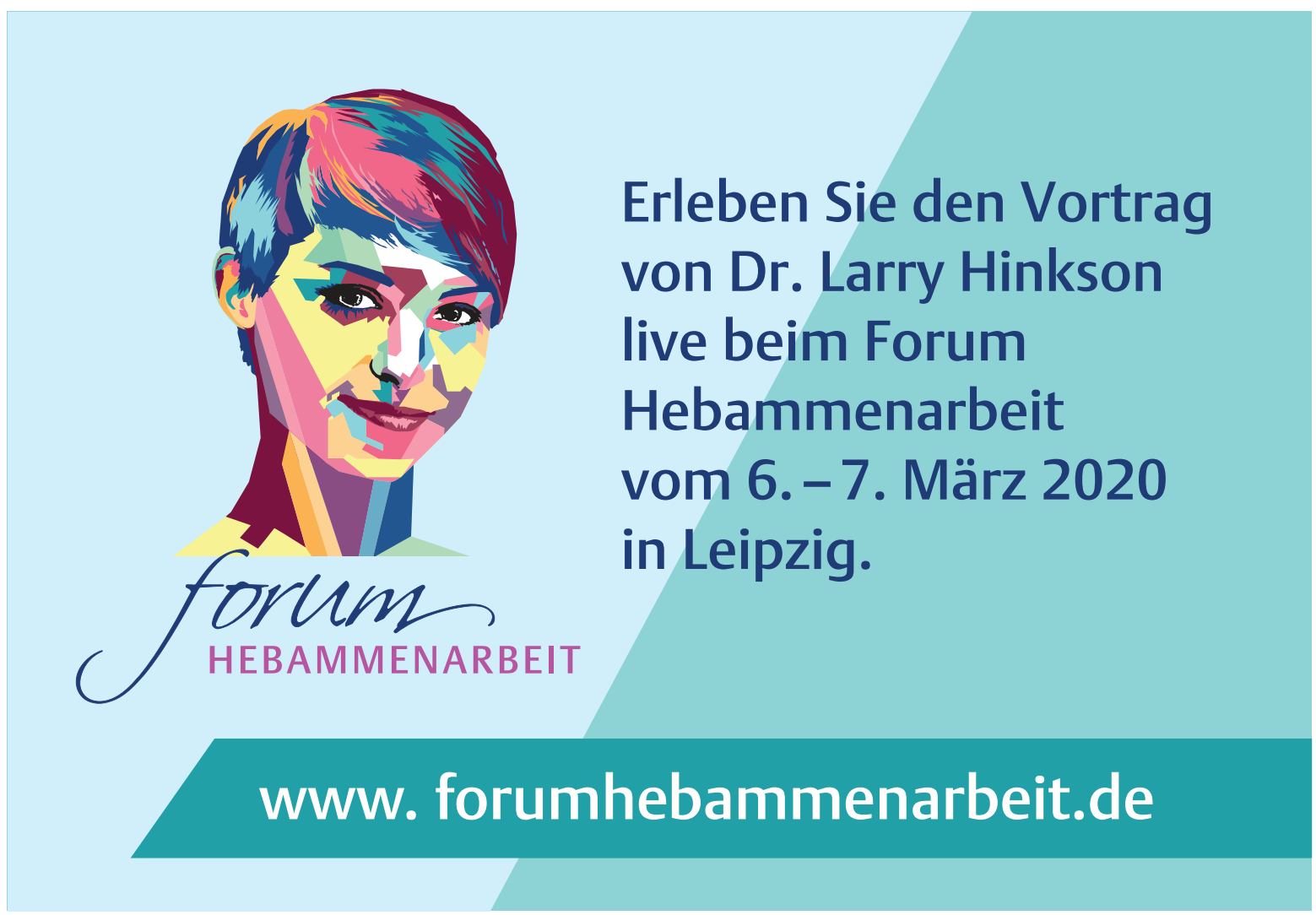

\section{Hintergrund}

Die äußere Wendung ist eine empfohlene Intervention mit geringem Risiko zur Behandlung der Beckenendlage ab der 36. SSW. Sie wird von internationalen Gesellschaften empfohlen, um die Wahrscheinlichkeit einer vaginalen Geburt zu verbessern. Die Erfolgsrate der äußeren Wendung variiert allerdings zwischen $35-86 \%$ [1].

Eine Arbeitsgruppe für Beckenendlage in der Charité Universitätsklinik Berlin erforscht die äußere Wendung zurzeit wissenschaftlich. Ziel ist es, die Erfolgsquote zu ermitteln und die günstigen Kriterien festzulegen, die mit einem erfolgreichen Wendungsversuch verbunden sind. Diese Ergebnisse sollen es ermöglichen, die Frauen bzw. Paare besser über die Erfolgschancen und das Risiko von Wendungen zu beraten sowie günstige und ungünstige Parameter zu ermitteln, damit Patientinnen informierte Entscheidungen treffen können.

\section{Sanfte äußere Wendung}

Oberarzt Dr. Larry Hinkson führt am Universitätsklinikum Charité Berlin eine viel beachtete sanfte äußere Wendung durch, für die er das sog. Soft-Touch-Verfahren entwickelt hat. Mithilfe einer speziellen Massagetechnik aktiviert er dabei die Eigenreflexe des in Beckenendlage befindlichen 
Kindes. Dieses wird dadurch angeregt, sich geleitet vom Behandler im Uterus selbst zu drehen. Hinkson konnte durch Anwendung der Methode die Erfolgsrate bei der äußeren Wendung von $40 \%$ auf $72 \%$ erhöhen.

Die äußere Wendung führt er nach den Leitlinien des Krankenhauses durch, die eine Voruntersuchung und einen Ultraschall ebenso erfordern wie die gründliche Aufklärung: Die Frau bekommt die Vorteile und die Risiken der Technik genau erläutert, um eine informierte Entscheidung treffen zu können. Sie gibt eine schriftliche Einverständniserklärung ab. Das Kind in BEL wird vor, während und nach dem Manöver überwacht, um dessen Wohlbefinden sicherzustellen. Ein Anästhesieteam und ein OP-Team stehen in Bereitschaft. Nach der erfolgreich durchgeführten Wendung bleibt die Schwangere ggf. eine Nacht zur Überwachung stationär, bevor sie mit der Aussicht auf eine normale vaginale Geburt die Klinik verlassen kann.

\section{Soft-Touch-Methode}

Die von Dr. Larry Hinkson entwickelte Soft-Touch-Methode ist eine besonders sanfte, nahezu schmerzfreie Form der äußeren Wendung. Sie nutzt natürliche frühkindliche Reflexe, die auch bei Neugeborenen zu beobachten und erforscht sind. Die Schwangere bekommt vor dem Manöver ein muskelentspannendes Mittel, jedoch ist währenddessen keine Tokolyse erforderlich. Alle Patientinnen werden routinemäßig bezüglich der Risiken, zum Beispiel mehrere Nabelschnurumschlingungen, beraten [2]. Die äußere Wendung wird von einem ausgebildeten Geburtshelfer mit Oberarztanwesenheit durchgeführt. Hier wird der Steiß manuell aus dem Becken gehoben unter Verwendung leichter Handbewegungen und Ausnutzen der natürlichen kindlichen Reflexe [3]. Das Kind dreht sich dann vorwärts oder rückwärts in Schädellage. Nach der Wendung wird ein CTG durchgeführt, um das Wohlbefinden

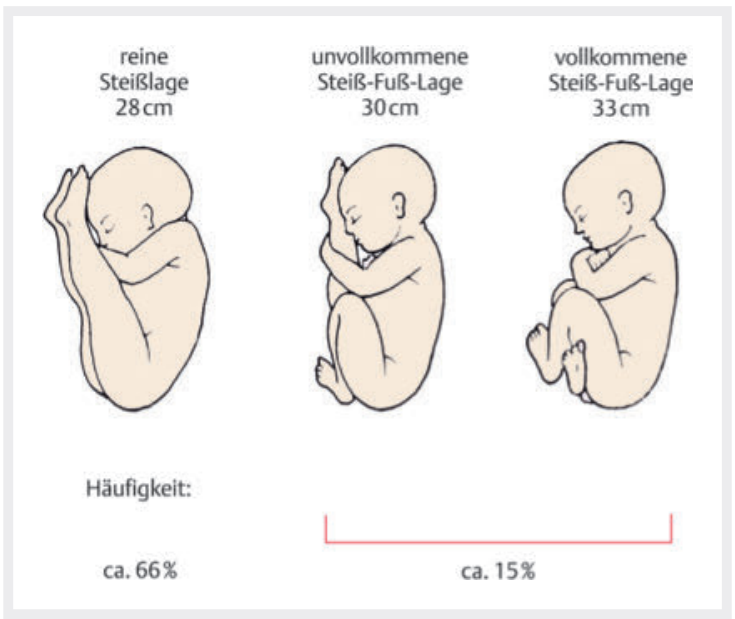

Abb. 1 Bei rund $70 \%$ der Kinder in Beckenendlage liegt eine reine Steißlage vor. (Quelle: Stiefel et al. Hebammenkunde [4]). des Kindes zu bestätigen. Vor der Entlassung wird auch eine Ultraschalluntersuchung durchgeführt.

\section{Fragen an den Experten}

Dr. Larry Hinkson berichtet auf dem Forum Hebammenarbeit 2020 vom aktuellen Stand der Arbeitsgruppe für Beckenendlage der Charité Universitätsklinik Berlin. Nach seinem Vortrag am 6. März 2020 in Leipzig und am 13. November 2020 in Wiesbaden besteht die Chance, Fragen zu stellen und zu diskutieren. Alle Infos zum Kongress unter www.forumhebammenarbeit.de

\section{Referent}

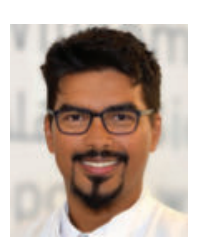

Dr. Larry Hinkson ist Leitender Oberarzt CCM an der Klinik für Geburtsmedizin der Charité Universitätsmedizin Berlin

\section{Korrespondenzadresse}

\author{
Charité - Universitätsmedizin Berlin \\ Klinik für Geburtsmedizin \\ Dr. Larry Hinkson \\ Charitéplatz 1 \\ 10117 Berlin \\ E-Mail: larry.hinkson@charite.de
}

\section{Literatur}

[1] Hofmeyr G], Kulier R. External cephalic version for breech presentation at term. Cochrane Database Syst Rev. 2000; (2) CD000083

[2] Hinkson L, Pahlitzsch T, Henrich W. Sextuple rings of nuchal cord by breech presentation: a warning sign.Ultrasound Obstet Gynecol. 2019 Jun 25. doi: 10.1002/uog.20376

[3] Hinkson L, Henrich W. Eliciting primitive fetal reflexes in the intrauterine environment: A new concept to aid external cephalic version. Eur J Obstet Gynecol Reprod Biol. 2018 Dec;231:283284. doi: 10.1016/j.ejogrb.2018.10.044. Epub 2018 Oct 25

[4] Stiefel A, Geist C, Harder U. Hebammenkunde. 5. Aufl. Thieme, Stuttgart 2013

\section{Bibliografie}

DOI https://doi.org/10.1055/a-1084-4975

Die Hebamme 2020; 33: 38-39

(c) Georg Thieme Verlag KG Stuttgart · New York

ISSN 0932-8122 